\title{
Identification of differentially expressed genes and regulatory relationships in Huntington's disease by bioinformatics analysis
}

\author{
XIAOYU DONG and SHUYAN CONG
}

\begin{abstract}
Department of Neurology, Shengjing Hospital of China Medical University, Shenyang, Liaoning 110004, P.R. China
\end{abstract}
Received September 27, 2017; Accepted December 20, 2017

DOI: $10.3892 / \mathrm{mmr} .2018 .8410$

\begin{abstract}
Huntington's disease (HD) is an inherited, progressive neurodegenerative disease caused by a CAG expansion in the huntingtin (HTT) gene; various dysfunctions of biological processes in HD have been proposed. However, at present the exact pathogenesis of HD is not fully understood. The present study aimed to explore the pathogenesis of HD using a computational bioinformatics analysis of gene expression. GSE11358 was downloaded from the Gene Expression Omnibus andthe differentially expressed genes (DEGs) in the mutant HTT knock-in cell model STHdhQ111/Q111 were predicted. DEGs between the HD and control samples were screened using the limma package in R. Functional and pathway enrichment analyses were conducted using the database for annotation, visualization and integrated discovery software. A protein-protein interaction (PPI) network was established by the search tool for the retrieval of interacting genes and visualized by Cytoscape. Module analysis of the PPI network was performed utilizing MCODE. A total of 471 DEGs were identified, including ribonuclease A family member 4 (RNASE4). In addition, 41 significantly enriched Kyoto Encyclopedia of Genes and Genomes pathways, as well as several significant Gene Ontology terms (including cytokine-cytokine receptor interaction and cytosolic DNA-sensing) were identified. A total of 18 significant modules were identified from the PPI network. Furthermore, a novel transcriptional regulatory relationship was identified, namely signal transducer and activator of transcription 3 (STAT3), which is regulated by miRNA-124 in HD. In conclusion, deregulation of 18 critical genes may contribute to the occurrence of HD. RNASE4, STAT3, and miRNA-124 may have a regulatory association with the pathological mechanisms in HD.
\end{abstract}

Correspondence to: Dr Shuyan Cong, Department of Neurology, Shengjing Hospital of China Medical University, 36 Sanhao Street, Shenyang, Liaoning 110004, P.R. China

E-mail: congsy_cmu@outlook.com

Key words: Huntington's disease, differentially expressed genes, functional and pathway enrichment analysis, protein-protein interaction network, microRNA

\section{Introduction}

Huntington's disease (HD) is a fatal neurodegenerative disease characterized by choreiform movements, personality changes, and dementia. HD is caused by a CAG trinucleotide expansion in exon 1 of the huntingtin gene (HTT) (1). Degenerative changes and cell death occur in extensive brain regions and outside the central nervous system (CNS), particularly involving the striatum; HD currently lacks effective treatment (2). As a consequence, HD results in cognitive and motor dysfunctions, involving speech, thought, psychiatric problems and involuntary muscle movements (3).

Over the years, studies on various pathological mechanisms including endoplasmic reticulum stress, oxidative stress, axonal transport, autophagy, excitotoxicity, mitochondrial function, the ubiquitin proteasome system (4), transcriptional deregulation and apoptosis have been implicated in HD (5). Among these, various molecular and cellular dysfunctions were shown to originate from mutant HTT (mHTT); transcriptional dysregulation is considered to be one of the most important events (6). Transcription factors (TFs) including CBP (7), p53 (8), Sp1 (9), NFkB, and TBP are recruited to aggregates formed by mHTT (10). Furthermore, HTT and mHTT are expressed in multiple tissues and can alter the transcription of miRNAs, such as miRNA-214, -150, $-146 \mathrm{a}$, and -137 , which have been shown to target the HTT gene (11).

Gene expression profile analysis is a fast, high-throughput method for detecting mRNA expression in tissues or cells. By comparing the different gene expression between HD models and healthy controls, a better understanding of the pathogenesis of HD can be acquired, facilitating the identification of potential target genes for therapy. Recent studies have suggested that bioinformatics mining and network analysis play an important role in studying and predicting the etiology in various neurodegenerative diseases, including HD (12-14).

The present study used the data from Sadri-Vakili et al (15) and the DEGs between STHdh111/111 and STHdh7/7 were identified. Possible functions were predicted using enrichment analysis; protein-protein interaction (PPI) networks were visualized and module analysis was conducted to screen for key genes in STHdh111/111. In addition, we predicted a new regulatory pathway involving miRNAs, TFs, and their target genes. We aimed to explore the pathogenesis of HD using a computational bioinformatics analysis of gene expression. 


\section{Materials and methods}

Affymetrix microarray data. Derivation of genetic data gene expression profile (GSE11358) was downloaded from a national center for biotechnology information GEO (http://www.ncbi. nlm.nih.gov/geo/) database. Experiments were designed to compare the changes of mRNA expression between wild and mutant HD mouse models by histone acetyltransferase inhibitor intervention. Four STHdh cell lines were used, expressing full-length versions of mutant 111 glutamines (STHdh111/111), along with four wild-type cell lines containing seven glutamines (STHdh7/7). The base data was built on the platform of GPL1261 and analyzed based on the affymetrix mouse genome 4302.0 array. In this study, GSE11358 was downloaded from a public database; therefore, patient consent ethics committee approval was not required.

Data pre-processing and analysis of DEGs. Original data was first converted into identifiable expression forms; the limma package (linear models for microarray data) in $\mathrm{R}$ language was used to identify DEGs between STHdh111/111 and STHdh7/7 (16). P-values of the DEGs were calculated separately and adjusted using the t-test method, and testing correction was performed using a Benjamini-Hochberg false discovery rate (HB FDR) (17), DEGs with FDR $<0.05$ and $1 \log$ fold change $(\mathrm{FC}) \mathrm{l}>2$ were used as thresholds.

Functional and pathway enrichment analysis. DAVID (database for annotation visualization and integrated discovery) online analysis tools constitute a comprehensive biological information database. The system can mine biological functions of a large number of genes and protein ID, and play a key role in further gene biological information extraction. Its website is http://david.abcc.ncifcrf.gov (18). Gene ontology database (GO; www.geneontology.org) depicts basic characteristics of genes and gene products (19). The Kyoto encyclopedia of genes and genomes (KEGG; www.genome. $\mathrm{jp} / \mathrm{kegg} /$ ) (20) pathway enrichment analysis was performed for identified DEGs using DAVID. Enriched terms with more than two genes and $\mathrm{P}$ values $<0.01$ were considered to be statistically significant.

Construction of a PPI network and analysis. The search tool for the retrieval of interacting genes (STRING http:/string. embl.de/) is an online database that has been designed as a comprehensive perspective to evaluate interaction information of proteins (21). In the present study, STRING was used to obtain a protein-protein interaction (PPI) network of DEGs, and subsequently visualized using Cytoscape (22). A confidence score of 0.4 was selected as the cut-off criterion. Molecular complex detection (MCODE) was then performed to screen modules of the PPI network with a degree cut-off $=2$, node score cut-off $=0.2, \mathrm{k}$-core $=2$, and $\max$, depth=100 (23). The functional enrichment analysis of genes was performed by DAVID in each module.

MicroRNAs prediction and transcription factor analysis. Biological targets of miRNAs were predicted by using TargetScan, which is one of the most commonly used bioinformatics target prediction tools (24). In the present study,

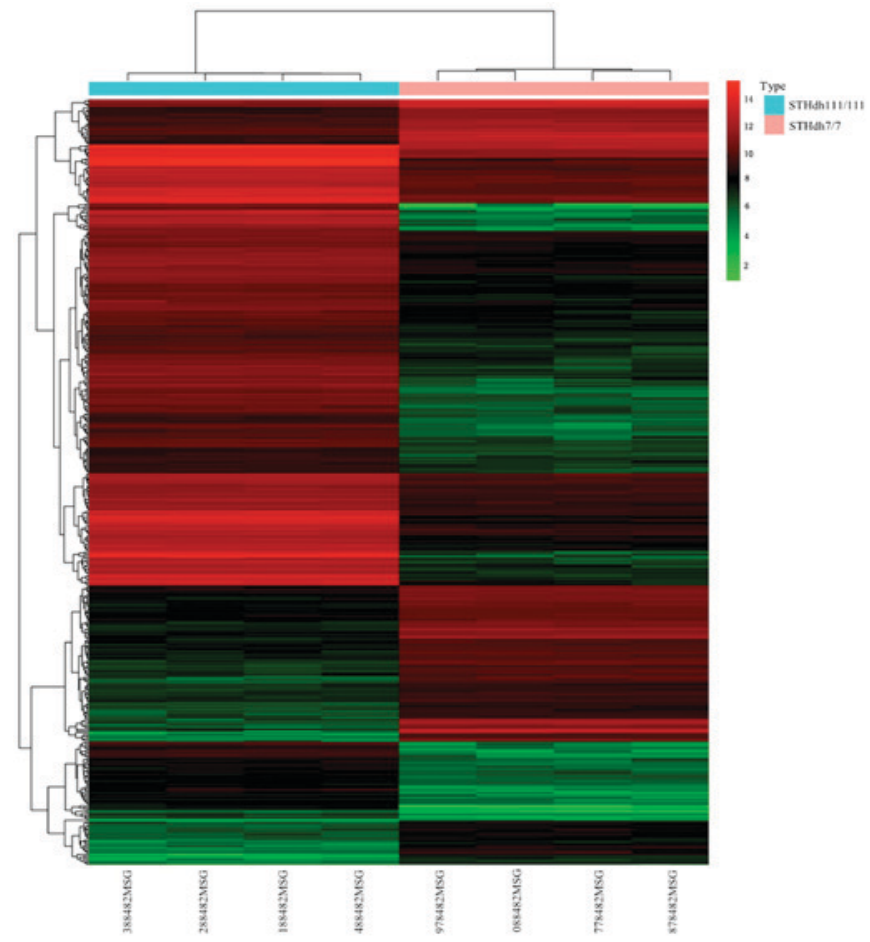

Figure 1. A cluster heat map of differentially expressed genes (DEGs). Red stands for high expression value, and black stands for low expression value. Changes of color from black to red represent the changes in expression value from low to high.

we chose the threshold of a region of 8mer seeds, which were completely matched for miRNA prediction. The TRANSFAC database is one of the most commonly used platform for the description and analysis of gene regulatory events and networks. It provides information about eukaryotic TFs, DNA-binding sites and DNA-binding profiles (25). In this study, we selected the TRANSFAC database for the description and prediction of TFs.

\section{Results}

Identification of DEGs. A total of 471 DEGs including 319 upregulated and 152 downregulated DEGs were selected. This set of DEGs was used for hierarchical clustering analysis (Fig. 1).

Functional and pathway enrichment analysis. Upregulated genes in the STHdh111/111 cells were significantly enriched in $208 \mathrm{GO}$ terms and $25 \mathrm{KEGG}$ pathways. The top ten functions enriched for upregulated genes are presented in Table I, including extracellular region $\left(\mathrm{P}=1.49 \times 10^{-28}\right)$ and immune system process $\left(\mathrm{P}=2.53 \times 10^{-19}\right)$. Downregulated genes in the STHdh111/111 cells were significantly enriched in $159 \mathrm{GO}$ terms and 16 KEGG pathways. The top ten functions enriched for downregulated genes are presented in Table II, including cell adhesion $\left(\mathrm{P}=5.06 \times 10^{-7}\right)$, protein binding $\left(\mathrm{P}=3.08 \times 10^{-7}\right)$, and cytoplasm $\left(\mathrm{P}=3.28 \times 10^{-6}\right)$.

Pathways enriched for upregulated genes included cytokine-cytokine receptor interaction $\left(\mathrm{P}=9.18 \times 10^{-8}\right)$, the cytosolic DNA-sensing pathway $\left(\mathrm{P}=3.39 \times 10^{-7}\right)$ and the Jak-STAT signaling pathway $\left(\mathrm{P}=5.15 \times 10^{-5}\right)$, presented in Table III. In 
Table I. Top 10 functions enriched for the upregulated genes in the STHdh111/111 cells.

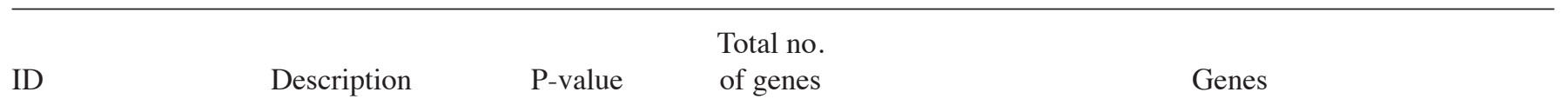

GO:0005576 Extracellular region $1.49 \times 10^{-28}$

93

GO:0009615 Response to virus $\quad 7.68 \times 10^{-23}$

GO:0051607

Defense response $2.82 \times 10^{-21}$ to virus

$\begin{array}{llll}\text { GO:0002376 } & \begin{array}{l}\text { Immune system } \\ \text { process }\end{array} & 2.53 \times 10^{-19} & 38 \\ & & \\ & & \\ \text { GO:0035458 } & \begin{array}{l}\text { Cellular response to } \\ \text { interferonxbeta }\end{array} & 4.27 \times 10^{-19} & 17 \\ & & \\ \text { GO:0045087 } & \begin{array}{l}\text { Innate immune } \\ \text { response }\end{array} & 5.67 \times 10^{-17} & 36\end{array}$

GO:0005615 Extracellular space $\quad 1.01 \times 10^{-16} \quad 68$
ASPN, AEBP1, FGF7, FAM3C, ARSJ, POSTN, LRRC17, MMP3, CXCL10, LOC100861978, NOV, OGN, C1RA, WISP2, C1RB, TNFRSF11B, CASP4, ISG15, SERPINE2, APOD, APOF, TGFBI, SEMA3E, RSPO2,CFH, BC028528,ANGPT1,SEMA3B, ITIH2, SEPP1, LBP, LOX, COL10A1, C4A, SPARCL1, ANG3, LIFR, MGP, NDNF, VEGFC, SERPINA3N, BGN, C4BP, SERPINA3M, ADM, EREG, COL1A2, ADAMTS1, ADAMTS5, HSD17B11, CXCL1, RBP4, IL1R1, WNT16, C3, ENPP2, LUM, CPQ, CLU, CXCL9, IL33, DCN, GBP2B, CCL5, ISLR, SMOC2, LGALS3BP, GLIPR1, FBN2, PTX3, COL8A2, THBS2, SVEP1, RNASE4, EFEMP1, IL1RN, IGF2, CLEC11A, LGALS9, GAS6, THSD7A, TSLP, OMD, LAMA4, SNED1, PENK, CLEC3B, CXCL15, TGFBR3, APOL9A, C1S1, IGFBP4, IGFBP5

IFIH1, BST2, CLU, OAS3, RSAD2, OAS2, CCL5, CXCL10, ISG20, DDX58, IFIT3, IFIT2, IFIT1, OASL2, DDX60, IFI27L2A, TGTP2, OAS1A, EIF2AK2, MX1, MX2, DHX58, DCLK1, ADAR IFIH1, SLFN9, CXCL9, OAS3, SLFN8, RSAD2, OAS2, IL33, GBP2B, CXCL10, ISG20, ISG15, OASL2, DDX60, MX1, MX2, DHX58, ZBP1, BST2, TRIM25, STAT2, H2xQ9, IFIT3, DDX58, IFIT2, IFIT1, OAS1A, EIF2AK2, ADAR

IFIH1, C3, OAS3, H2xD1, RSAD2, OAS2, GBP2B, ISG20, C1RA, C1RB, CASP4, OASL2, TAP1, CFH, IIGP1, LBP, MX1, MX2, DHX58, ZBP1, H2xK1, IRGM1, H2xL, BST2, HERC6, TRIM25, PSMB8, LGALS9, PSMB9, IFIT3, DDX58, IFIT2, IFIT1, C4BP, IRF7, C1S1, EIF2AK2, ADAR

GBP6, IRGM1, IRGM2, LOC100044068, IFI47,

STAT1, GBP2B, IFIT3, IFI202B, IFIT1, IGTP, IIGP1, TGTP2, GBP3, GM4951, GBP2, IFI204

IFIH1, C3, TRIM14, OAS3, RSAD2, OAS2, ISG20, C1RA, C1RB, CASP4, OASL2, CFH, IIGP1, VNN1, LBP, PTX3, MX1, MX2, DHX58, ZBP1, IRGM1, LOC100044068, BST2, HERC6, TRIM25, IFI202B, DDX58, IFIT3, IFIT2, IFIT1, C4BP, IRF7, OAS1A, C1S1, EIF2AK2, ADAR

AEBP1, FGF7, POSTN, LRRC17, DLK1, CXCL11, MMP3, CXCL10, LOC100861978, OGN, TNFRSF11B, WISP2, SERPINE2, APOD, SEMA3E, TGFBI, CFH, VNN1, ANGPT1, SEMA3B, CES1D, SEPP1, LOX, LBP, CTSZ, C4A, SPARCL1, MGP, VEGFC, SERPINA3N, THBD, SERPINA3M, ADM, EREG, PPBP, COL1A2, CTSH, ADAMTS5, CXCL1, RBP4, WNT16, IL1R1, ENPP2, C3, LUM, CPQ, CLU, CXCL9, OAS3, IL33, DCN, CCL5, ABI3BP, LGALS3BP, COL6A3, PTX3, EFEMP1, IL1RN, LMCD1, IGF2, GAS6, CLEC11A, TSLP, OMD, CLEC3B, CXCL15, TGFBR3, IGFBP4 
Table I. Continued.

\begin{tabular}{|c|c|c|c|c|}
\hline ID & Description & P-value & $\begin{array}{l}\text { Total no. } \\
\text { of genes }\end{array}$ & Genes \\
\hline GO:0005578 & $\begin{array}{l}\text { Proteinaceous } \\
\text { extracellular matrix }\end{array}$ & $5.89 \times 10^{-16}$ & 31 & $\begin{array}{l}\text { ASPN, WNT16, LUM, POSTN, DCN, MMP3, } \\
\text { ABI3BP, NOV, SMOC2, OGN, TNFRSF11B, } \\
\text { LGALS3BP, WISP2, TGFBI, COL6A3, FBN2, LOX, } \\
\text { THBS2, COL8A2, COL10A1, SPARCL1, EFEMP1, } \\
\text { NDNF, LAMA4, OMD, BGN, CLEC3B, COL1A2, } \\
\text { TGFBR3, ADAMTS1, ADAMTS5 }\end{array}$ \\
\hline GO:0008201 & Heparin binding & $1.79 \times 10^{-12}$ & 20 & $\begin{array}{l}\text { FGF7, POSTN, CCL5, CXCL11, ABI3BP, NDNF, } \\
\text { CXCL10, NOV, SMOC2, OGN, WISP2, SERPINE2, } \\
\text { CLEC3B, RSPO2, CFH, TGFBR3, ADAMTS1, } \\
\text { GPNMB, THBS2, ADAMTS5 }\end{array}$ \\
\hline GO:0031012 & Extracellular matrix & $1.32 \times 10^{-11}$ & 25 & $\begin{array}{l}\text { ASPN, AEBP1, LUM, CLU, POSTN, DCN, MMP3, } \\
\text { NOV, OGN, LGALS3BP, CD93, SERPINE2, } \\
\text { COL6A3, TGFBI, FBN2, COL8A2, THBS2, } \\
\text { EFEMP1, LMCD1, MGP, OMD, BGN, COL1A2, } \\
\text { ADAMTS1, ADAMTS5 }\end{array}$ \\
\hline
\end{tabular}

addition, downregulated genes were significantly enriched in the p53 signaling pathway $\left(\mathrm{P}=2.23 \times 10^{-3}\right)$, the regulation of actin cytoskeleton $(\mathrm{P}=0.032)$ and the hippo signaling pathway $(\mathrm{P}=0.036)$, presented in Table IV.

PPI network construction and analysis. The PPI network of DEGs was constructed by Cytoscape software following a PPI search (Fig. 2). Using the MCODE in Cytoscape, degrees $\geq 10$ was set as the cutoff criterion and a total of 18 genes were selected as hub genes (DHX58, HERC6, RTP4, IFI44, STAT2, MX2, STAT1, IRF9, CXCL10, IFIT2, USP18, ISG15, OASL2, IFIT1, RSAD2, IRF7, DDX58, and IFIT3) were identified from the PPI network (Fig. 3). Notably, there were higher degrees in IFIT1 and OASL2 (degree $=20)$ in the PPI network for upregulated genes, and in DDX58 and HERC6 (degree=20) in the PPI network for downregulated genes.

miRNA prediction of DEGs. From the 471 DEGs investigated by this study, we chose a DEG with the largest log fold change (FC) value for miRNA prediction and analysis, which was RNASE4. We mapped the RNASE4 $(\log \mathrm{FC}=9.11, \mathrm{P}$ Value $=1.32 \times 10^{-07}$ ) in the TargetScan database for predicting the miRNAs of this gene. By choosing the strict cutoff of 8mer seeds, we found two miRNAs: miRNA-124a and miRNA-124a2.

Transcription factor prediction of DEG. We searched the TFs of the RNASE4 gene by using the TRANSFAC database. Ten TFs were identified, including nuclear factor (NF)- $\mathrm{BB}$, c-Jun, c-Fos, hepatocyte nuclear factor (HNF)-4 $\alpha 1$, HNF-4 $\alpha 2$, activating protein-1 (AP-1), myocyte enhancer binding factor 2 (aMEF-2), signal transducer and activator of transcription 3 (STAT3), and cAMP-response element binding protein (SCREB; Fig. 4). The image displays the most relevant transcription factor binding sites in the promoter region of this gene as predicted by TRANSFAC database (25). We predicted the miRNAs of these identified TFs and found that only STAT3 was associated with miRNA-124.

\section{Discussion}

In this study, bioinformatics analysis of GSE11358 was carried out; we identified a total of 471 DEGs in STHdh111/111 cells compared with normal STHdh7/7 cells, including 319 upregulated and 152 downregulated genes. Upon analysis by MCODE in Cytoscape, 18 modules were identified from the PPI network, including DHX58, HERC6, RTP4, IFI44, STAT2, MX2, STAT 1, IRF9, CXCL10, IFIT2, USP18, ISG15, OASL2, IFIT1, RSAD2, IRF7, DDX58, and IFIT3. Of these, IFIT1 and OASL2 had the highest degree in upregulated genes, while DDX58 and HERC6 had the highest degree in downregulated genes.

IFIT1 was identified as a member of a macrophage 'core response module', which was commonly differentially expressed in response to multiple stimulatory signals in the immune system (26). Interestingly, previous studies have demonstrated that both innate and adaptive immune systems were activated during progression of HD (27). Dysregulation of IFIT1 can cause a measurable effect in the expression of downstream targets, acting either directly or indirectly, and IFIT1 was associated with a cluster of genes related to the innate immune response. Bayram-Weston et al found that IFIT1 was highly expressed in the striatum of the YAC128 and HdhQ150 mouse models of Huntington's disease (28); Jordanovski et al also found that high expression of IFIT1 was correlated with Huntington's disease in the study of the TF ZNF395 (29). All these results suggested that IFIT1 plays an important role in the immune response by regulating macrophage function, and its abnormal regulation could be involved in the pathophysiological changes of HD. However, the exact regulatory mechanism is unclear, and further experiments are needed to define the mechanism 
Table II. Top 10 functions enriched for the down regulated genes in the STHdh111/111 cells.

\begin{tabular}{|c|c|c|c|c|}
\hline ID & Description & P-value & $\begin{array}{l}\text { Total no. } \\
\text { of genes }\end{array}$ & Genes \\
\hline GO:0007155 & Cell adhesion & $5.06 \times 10^{-7}$ & 17 & $\begin{array}{l}\text { FLRT2, CADM1, NUAK1, PDPN, ITGB5, ITGA3, } \\
\text { NECTIN4, CDH2, MEGF10, NCAM1, HES1, } \\
\text { WISP1, PKP1, CD34, TENM3, ITGA7, HAS2 }\end{array}$ \\
\hline GO:0005515 & Protein binding & $3.08 \times 10^{-7}$ & 56 & $\begin{array}{l}\text { ALDH1L1, CADM1, ATL2, AQP5, PAX6, PMAIP1, } \\
\text { PRKG2, ANKRD1, CKB, WNT4, UNC5B, TIAM1, } \\
\text { BOK, EMID1, PID1, KIF5C, BASP1, ECT2, } \\
\text { HES1, NCAM1, KRT19, UHRF1, SIX1, BUB1B, } \\
\text { WNT9A, KIF26B, MAP3K11, SOX2, BEX1, } \\
\text { CDH2, SOX6, CEP55, IVNS1ABP, PEX5L, VDR, } \\
\text { LHX2, POU3F3, SCN5A, OLFM1, DTNA, FLRT2, } \\
\text { NES, TRPC6, IGF1, BIRC5, DPYSL3, ITGA3, } \\
\text { SOD3, NREP, BMPER, PKP1, SFRP2, SALL1, } \\
\text { ITGA7, ID4, FCGBP }\end{array}$ \\
\hline GO:0005737 & Cytoplasm & $3.28 \times 10^{-6}$ & 75 & $\begin{array}{l}\text { ALDH1L1, CRABP1, PTGS2, TUBB2B, NUAK1, } \\
\text { CRABP2, PTGS1, PAX6, RPRM, ANKRD1, CKB, } \\
\text { WNT4, WISP1, BOK, TIAM1, PID1, CDC6, } \\
\text { SGOL1, KIF5C, BASP1, UBE2C, ECT2, HES1, } \\
\text { TNNT2, NCAM1, PPM1E, TAGLN, SPAG5, CD34, } \\
\text { SIX1, BUB1B, HAS2, FILIP1L, GRB14, CASQ2, } \\
\text { KIF26B, MAP3K11, FHL1, SOX2, DIAPH3, } \\
\text { BEX1, ANLN, CDH2, DENND2A, CEP55, } \\
\text { SERPINB1B, IVNS1ABP, PEX5L, TK1, ACSBG1, } \\
\text { NCAPG, FNDC1, ENO3, DTNA, FLRT2, PTGR1, } \\
\text { NES, GSTA4, TRPC6, IGF1, BIRC5, DPYSL3, } \\
\text { C330027C09RIK, RGS16, SOD3, CENPI, } \\
\text { FAM64A, NREP, SALL1, PRKAR1B, ITGA7, ID4, } \\
\text { FAM84B, BTBD11, PLEKHA1 }\end{array}$ \\
\hline GO:0000902 & Cell morphogenesis & $2.80 \times 10^{-5}$ & 7 & $\begin{array}{l}\text { VDR, CAP2, PDPN, TENM3, SOX6, GREM1, } \\
\text { ECT2 }\end{array}$ \\
\hline GO:0051301 & Cell division & $9.48 \times 10^{-5}$ & 12 & $\begin{array}{l}\text { CCNE2, CDC6, FAM64A, SPAG5, SGOL1, NUF2, } \\
\text { BUB1B, BIRC5, ANLN, CEP55, UBE2C, ECT2 }\end{array}$ \\
\hline GO:0007275 & $\begin{array}{l}\text { Multicellular organism } \\
\text { development }\end{array}$ & $1.72 \times 10^{-4}$ & 20 & $\begin{array}{l}\text { FLRT2, NES, CADM1, PDPN, FHL1, } \\
\text { SOX2, PAX6, BEX1, SOX6, SHOX2, VDR, WNT4, } \\
\text { UNC5B, SFRP2, SEMA7A, SIX1, POU3F3, } \\
\text { WNT9A, OLFM1, KIF26B }\end{array}$ \\
\hline GO:0045165 & Cell fate commitment & $1.82 \times 10^{-4}$ & 6 & HES1, WNT4, SOX2, PAX6, SOX6, WNT9A \\
\hline GO:0007067 & Mitotic nuclear division & $2.00 \times 10^{-4}$ & 10 & $\begin{array}{l}\text { CDC6, FAM64A, SPAG5, SGOL1, NUF2, BUB1B, } \\
\text { BIRC5, ANLN, CEP55, UBE2C }\end{array}$ \\
\hline GO:0008283 & Cell proliferation & $2.14 \times 10^{-4}$ & 9 & $\begin{array}{l}\text { UHRF1, PDPN, BOK, CD34, PRKAR1B, SIX1, } \\
\text { IGF1, ID4, MAP3K11 }\end{array}$ \\
\hline GO:0005911 & Cell-cell junction & $6.26 \times 10^{-4}$ & 8 & $\begin{array}{l}\text { NCAM1, FLRT2, CADM1, TIAM1, FNDC1, } \\
\text { CDH2, NECTIN4, ECT2 }\end{array}$ \\
\hline
\end{tabular}

in HD. OASL2, as an interferon-stimulated gene is another gene related to the innate immune response (30), and was shown to be an important response module in macrophage activation (31). In the BACHD mouse model of HD, impaired migration of macrophages in response to an inflammatory stimulus was shown, which predicted one of the underlying mechanisms of HD (32). McDermott et al reported that
OASL2 participates in interactions that are important for macrophage activation and migration (33). Dysregulation of OASL2 may interrupt migration of macrophages in the HD mice model or interact with mHTT fragments in their immunological functions.

DDX58, also known as retinoic acid inducible gene-I (RIG-I), has been validated by immunofluorescence labeling 
Table III. Top 10 enriched pathways for the upregulated genes.

\begin{tabular}{|c|c|c|c|c|}
\hline ID & Description & P-value & $\begin{array}{l}\text { Total no. } \\
\text { of genes }\end{array}$ & Genes \\
\hline $\mathrm{mmu} 05168$ & Herpes simplex infection & $1.70 \times 10^{-11}$ & 21 & $\begin{array}{l}\text { H2-K1, IFIH1, SP100, SOCS3, C3, } \\
\text { OAS3, H2-D1, OAS2, STAT1, CCL5, } \\
\text { H2-Q6, STAT2, IRF9, DDX58, IKBKE, } \\
\text { IFIT1, IRF7, TAP1, LOC101056305, } \\
\text { OAS1A, EIF2AK2 }\end{array}$ \\
\hline $\mathrm{mmu} 05164$ & Influenza A & $4.28 \times 10^{-11}$ & 19 & $\begin{array}{l}\text { IFIH1, SOCS3, OAS3, RSAD2, TRIM25, } \\
\text { IL33, OAS2, STAT1, CCL5, STAT2, } \\
\text { CXCL10, IRF9, DDX58, IKBKE, IRF7, } \\
\text { OAS1A, EIF2AK2, MX2, ADAR }\end{array}$ \\
\hline mmu04060 & $\begin{array}{l}\text { Cytokine-cytokine } \\
\text { receptor interaction }\end{array}$ & $9.18 \times 10^{-8}$ & 18 & $\begin{array}{l}\text { IL1R1, LTBR, OSMR, IL6ST, LIFR, } \\
\text { CXCL9, ACKR3, CCL5, CXCL11, } \\
\text { CXCL10, LOC100861969, } \\
\text { LOC100861978, VEGFC, TSLP, } \\
\text { TNFRSF11B, PPBP, CXCL15, IL13RA1 }\end{array}$ \\
\hline mmu04623 & $\begin{array}{l}\text { Cytosolic DNA- } \\
\text { sensing pathway }\end{array}$ & $3.39 \times 10^{-7}$ & 10 & $\begin{array}{l}\text { IFI202B, DDX58, IKBKE, } \\
\text { LOC100044068, IRF7, IL33, CCL5, } \\
\text { ZBP1, ADAR, CXCL10 }\end{array}$ \\
\hline mmu05160 & Hepatitis C & $6.55 \times 10^{-7}$ & 13 & $\begin{array}{l}\text { SOCS3, OAS3, OAS2, STAT1, STAT2, } \\
\text { IRF9, DDX58, IKBKE, IFIT1, IRF7, } \\
\text { CLDN1, OAS1A, EIF2AK2 }\end{array}$ \\
\hline mmu05162 & Measles & $6.55 \times 10^{-7}$ & 13 & $\begin{array}{l}\text { IFIH1, OAS3, OAS2, STAT1, STAT2, } \\
\text { IRF9, DDX58, IKBKE, IRF7, OAS1A, } \\
\text { EIF2AK2, MX2, ADAR }\end{array}$ \\
\hline mmu04630 & Jak-STAT signaling pathway & $5.15 \times 10^{-5}$ & 11 & $\begin{array}{l}\text { STAT6, IRF9, TSLP, OSMR, IL6ST, } \\
\text { SOCS3, LIFR, STAT1, IL13RA1, } \\
\text { STAT2, LOC100861969 }\end{array}$ \\
\hline $\mathrm{mmu} 04622$ & $\begin{array}{l}\text { RIG-I-like receptor signaling } \\
\text { pathway }\end{array}$ & $5.87 \times 10^{-5}$ & 8 & $\begin{array}{l}\text { DDX58, IKBKE, IFIH1, ISG15, IRF7, } \\
\text { TRIM25, DHX58, CXCL10 }\end{array}$ \\
\hline mmu04620 & $\begin{array}{l}\text { Toll-like receptor signaling } \\
\text { pathway }\end{array}$ & $6.98 \times 10^{-4}$ & 8 & $\begin{array}{l}\text { IKBKE, IRF7, CXCL9, LBP, STAT1, } \\
\text { CCL5, CXCL11, CXCL10 }\end{array}$ \\
\hline mmu05203 & Viral carcinogenesis & $2.17 \times 10^{-3}$ & 11 & $\begin{array}{l}\text { H2-K1, IRF9, LTBR, SP100, IL6ST, } \\
\text { C3, IRF7, H2-D1, LOC101056305, } \\
\text { EIF2AK2, H2-Q6 }\end{array}$ \\
\hline
\end{tabular}

of motor neurons in an amyotrophic lateral sclerosis mouse(TDP-43A315T) spinal cord (34). DDX58 is a direct target of TDP-43, and abnormalities of TDP-43 were shown to lead to deregulation of DDX58. Tauffenberger et al confirmed the results that inappropriate cytoplasmic accumulations of TDP-43 are observed in HD (35). In our study, we found that DDX58 was clearly downregulated, which inferred that abnormal accumulations of TDP-43 in HD could be responsible for this result. Other studies also found that DDX58 was involved in immune regulation (36).

HERC6 is a HerC protein family member; a previous study found that HERC6 was the main E3 ligase for global ISG15 conjugation in mouse cells (37). ISG15, an interferon (IFN)-stimulated gene, was reported to participate in activation of IFN signaling in the CNS and was usually associated with inflammation (38). Together, some studies indicated that ISG15 could activate autophagy by various means (39).
Although there were no exact studies reporting a correlation between HERC6 and HD, it has been established that aberrant inflammation and autophagy may both participate in the pathogenesis of HD; therefore, we believe that HERC6 may play a role in the etiology of HD by influencing the function of ISG15.

KEGG pathways, in our present study, including the Jak-STAT signaling pathway (37), the toll-like receptor signaling pathway $(13,40)$, and the RIG-I-like receptor signaling pathway (41) have been confirmed to be associated with HD and neurodegenerative diseases. Although we did not explore these pathways further in the present study, they are critical for understanding the pathogenesis of HD.

MicroRNA (miRNA) is a small non-coding RNA molecule containing about 22 nucleotides, and mediates the post-transcriptional regulation of gene expression (42). It has been reported that miRNA is abundantly expressed in the 
Table IV. Top 10 enriched pathways for the downregulated genes.

\begin{tabular}{|c|c|c|c|c|}
\hline ID & Description & P-value & $\begin{array}{l}\text { Total no. } \\
\text { of genes }\end{array}$ & Genes \\
\hline mmu05414 & Dilated cardiomyopathy & $5.90 \times 10^{-4}$ & 6 & $\begin{array}{l}\text { TNNT2, ADCY7, ITGA7, IGF1, } \\
\text { ITGB5, ITGA3 }\end{array}$ \\
\hline mmu04550 & $\begin{array}{l}\text { Signaling pathways regulating } \\
\text { pluripotency of stem cells }\end{array}$ & $9.37 \times 10^{-4}$ & 7 & $\begin{array}{l}\text { INHBB, WNT4, SOX2, PAX6, IGF1, } \\
\text { ID4, WNT9A }\end{array}$ \\
\hline mmu04923 & Regulation of lipolysis in adipocytes & $1.22 \times 10^{-3}$ & 5 & $\begin{array}{l}\text { ADCY7, PTGS2, PTGS1, PDE3B, } \\
\text { PRKG2 }\end{array}$ \\
\hline mmu04115 & p53 signaling pathway & $2.23 \times 10^{-3}$ & 5 & $\begin{array}{l}\text { CCNE2, RPRM, IGF1, PMAIP1, } \\
\text { IGFBP3 }\end{array}$ \\
\hline mmu05412 & $\begin{array}{l}\text { Arrhythmogenic right ventricular } \\
\text { cardiomyopathy (ARVC) }\end{array}$ & $2.75 \times 10^{-3}$ & 5 & TCF7, ITGA7, ITGB5, ITGA3, CDH2 \\
\hline mmu05410 & Hypertrophic cardiomyopathy (HCM) & $4.05 \times 10^{-3}$ & 5 & $\begin{array}{l}\text { TNNT2, ITGA7, IGF1, ITGB5, } \\
\text { ITGA3 }\end{array}$ \\
\hline mmu05200 & Pathways in cancer & 0.016 & 9 & 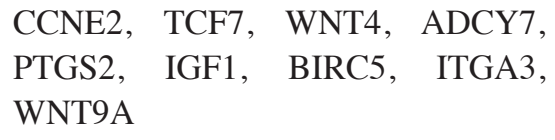 \\
\hline mmu05205 & Proteoglycans in cancer & 0.026 & 6 & $\begin{array}{l}\text { WNT4, TIAM1, IGF1, ITGB5, } \\
\text { WNT9A, GPC1 }\end{array}$ \\
\hline $\mathrm{mmu} 04810$ & Regulation of actin cytoskeleton & 0.032 & 6 & $\begin{array}{l}\text { TIAM1, ITGA7, DIAPH3, ITGB5, } \\
\text { ITGA3, MYL9 }\end{array}$ \\
\hline mmu04390 & Hippo signaling pathway & 0.036 & 5 & $\begin{array}{l}\text { TCF7, WNT4, SOX2, } \text { BIRC5, } \\
\text { WNT9A }\end{array}$ \\
\hline
\end{tabular}

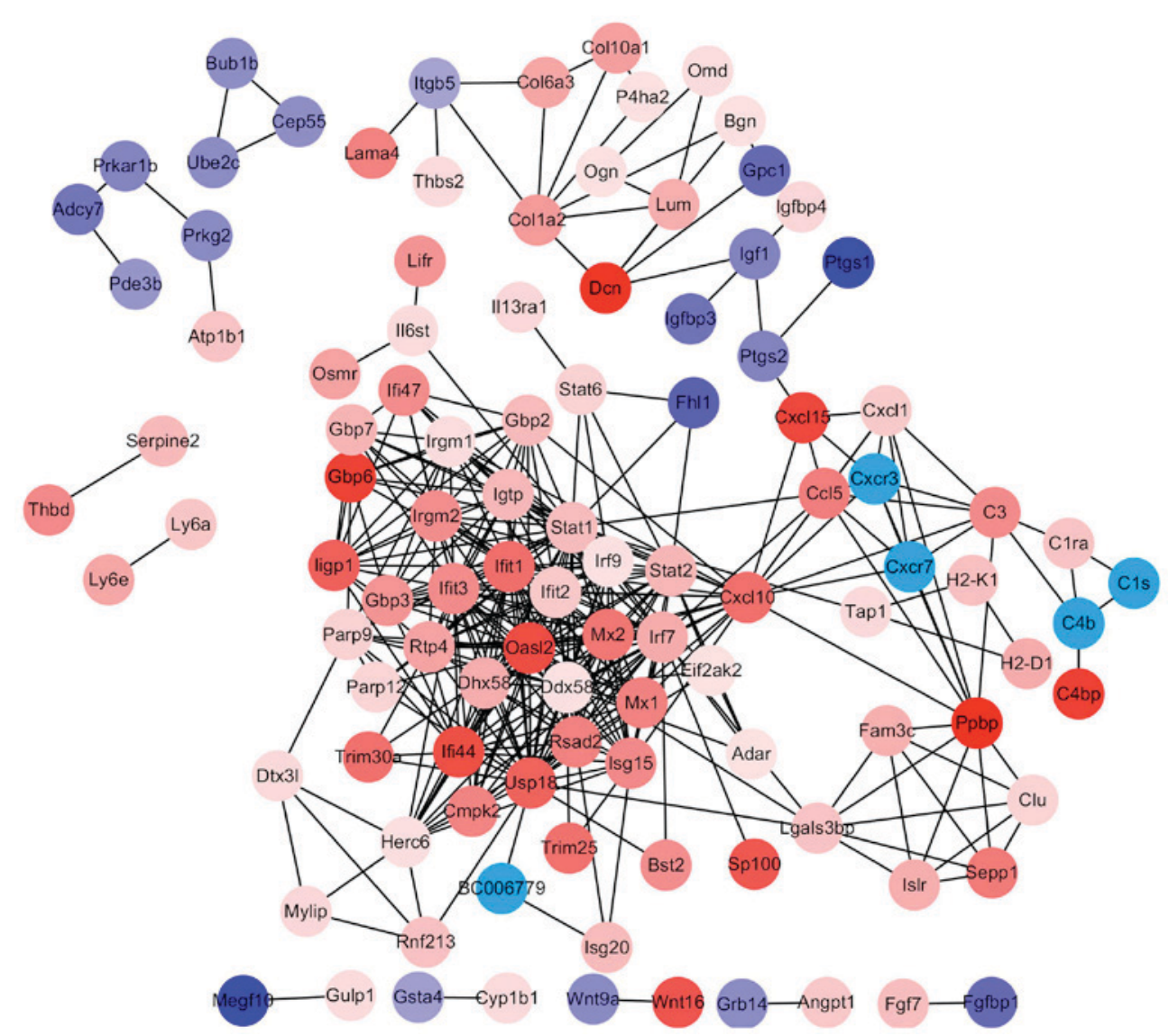

Figure 2. Protein-protein interaction network constructed for the DEGs. Red stands for high expression value and blue stands for low expression value. Changes of color from blue to red represent the changes in expression value from low to high. 


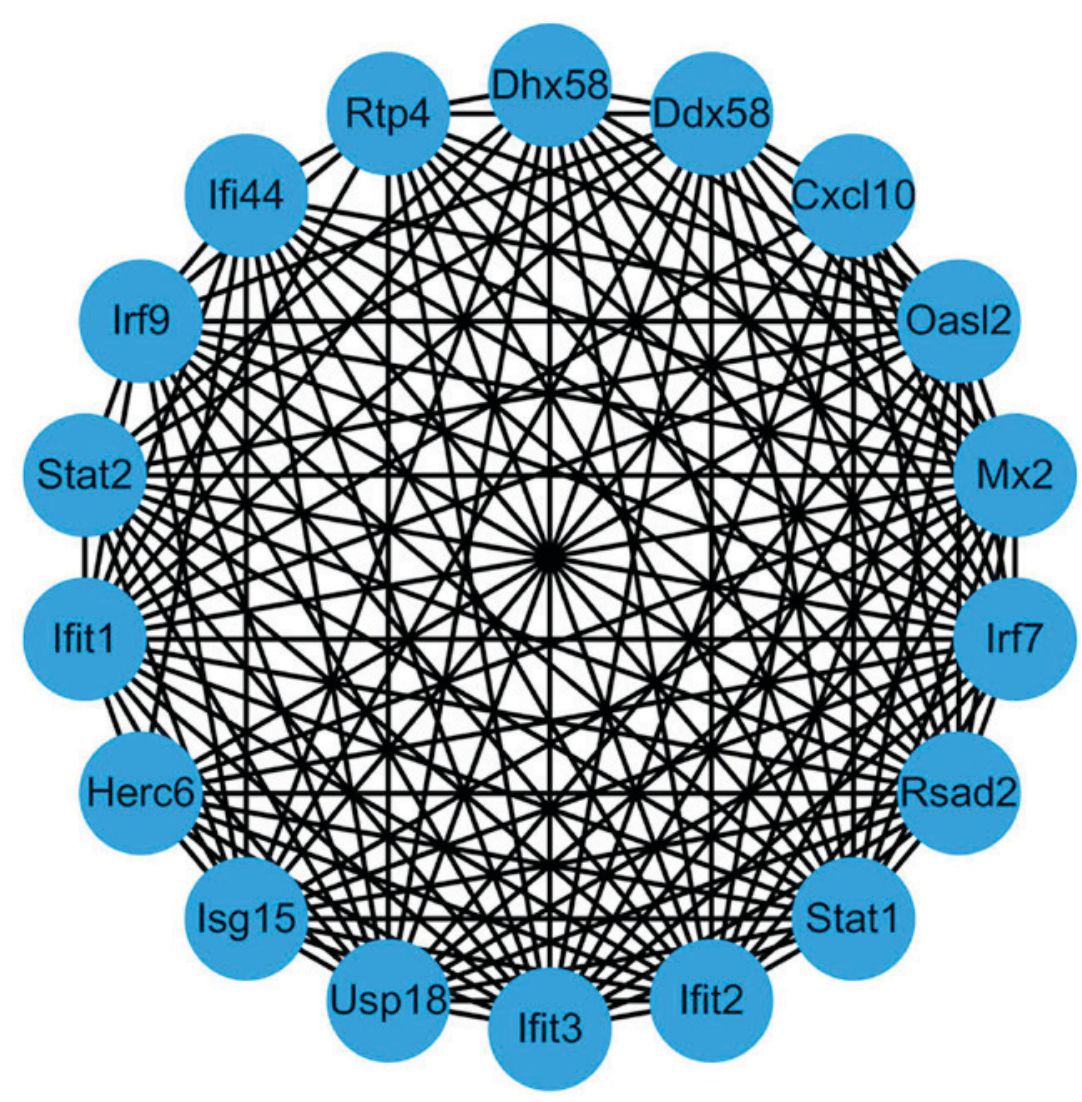

Figure 3. A significant module selected from the protein-protein interaction network by utilizing MCODE in Cytoscape. Lines represent an interacting relationship between nodes.

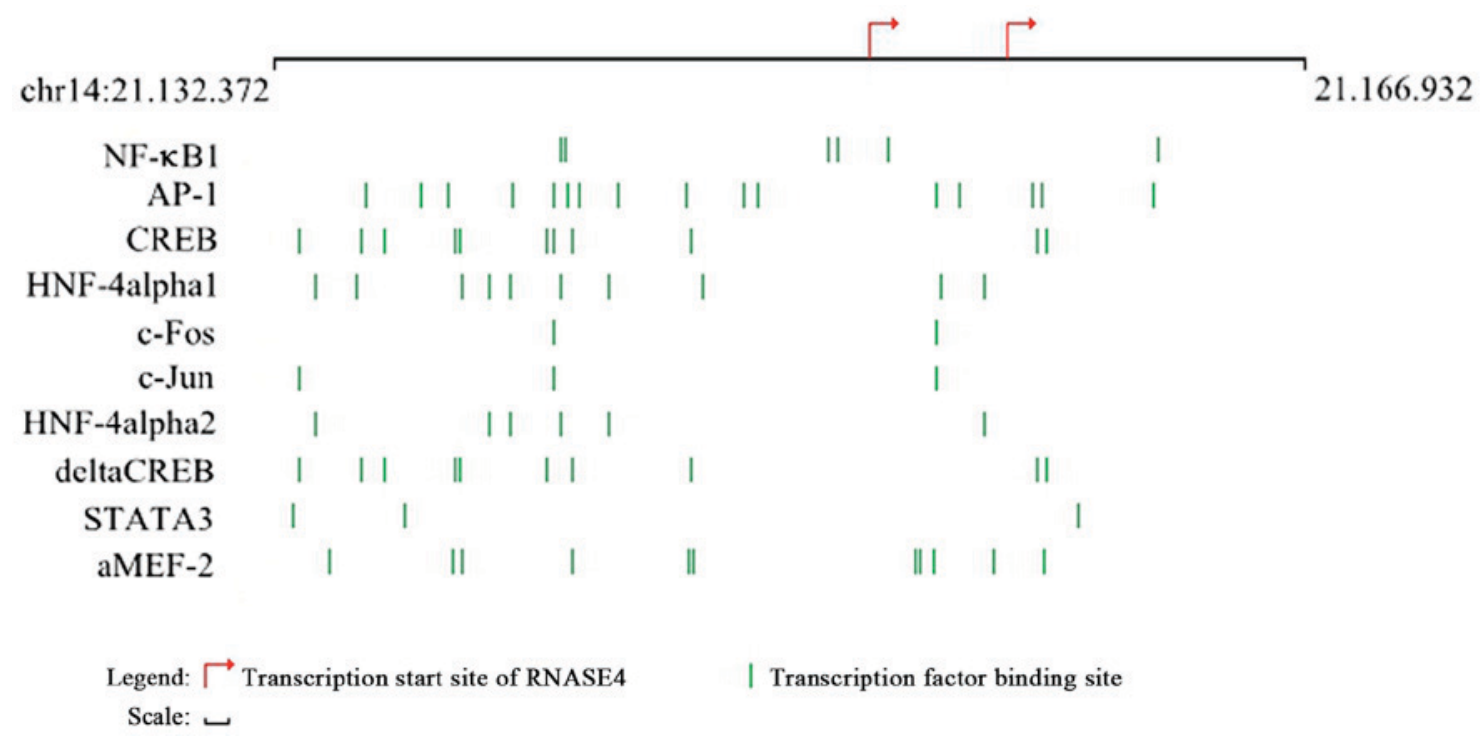

Figure 4. Transcription factors of RNASE4 gene in the TRANSFAC database. The red arrow indicates the transcription starting site and direction of the RNASE4 gene. The green vertical lines indicate the transcription factor binding sites. NF, nuclear factor; CREB, cAMP-response element binding protein; HNF, hepatocyte nuclear factor; AP, activating protein; aMEF, myocyte enhancer binding factor; STAT3, signal transducer and activator of transcription 3; delta CREB, cAMP-response element binding protein.

CNS (43) and may participate in diverse biological processes in neuronal cell differentiation and functions (44). A number of different miRNAs have been found to be abnormally regulated in cellular and mice models of HD, including miRNA-137, -214 (45), -146a (46), and -27a (47). Therefore, deregulated miRNAs could be attributed in etiology and therapeutic targets in the HD. Here, we tried to predict the miRNAs and TFs of DEGs in the HD mice model. We chose ribonuclease 4 (RNASE4), which is most significantly upregulated in HD ( $\log \mathrm{FC}=9.11)$. RNASE4, the fourth member of this superfamily, shares the same promoters as angiogenin (ANG), and is co-expressed with ANG (48). It has been reported that 
RNASE4 protects neuron degeneration by promoting angiogenesis, neurogenesis, and neuronal survival under stress (49). The neuroprotective activity of RNASE4 is similar to that of ANG, which can upregulate Bcl-2 (50) and inhibit nuclear translocation of apoptosis-inducing factor (51).

To strictly predict the miRNAs of the target gene RNASE4, we chose an exact match (8mer seed) to positions $2-8$ of the mature miRNA. We found that miRNA-124 had the highest score, suggesting that miRNA-124 could play an important role in HD, which was in line with previous studies (52). MicroRNA-124 has been implicated in HD by a mechanism that involves the regulation of CCNA2, and is involved in regulating the cell cycle (53).

By searching TRANSFAC databases, we found STAT3 was also regulated by miRNA-124. Astrocyte reactivity is a hallmark of neurodegenerative diseases (ND); activation of the JAK/STAT3 pathway can promote astrocyte reactivity and decrease the number of huntington aggregates, a neuropathological hallmark of HD (54). By analyzing new regulatory relationships between miRNA-124, STAT3 and RNASE4, we predicted a novel regulatory pathway that miRNA-124 regulates TF STAT3, and TF STAT3 regulates RNASE4, that is, miRNA-124 $\rightarrow$ STAT3 $\rightarrow$ RNASE4, which could be a possible target for HD treatment.

In the present study, we investigated the underlying mechanisms of HD via bioinformatics analysis. A total 471 DEGs were identified, and 18 hub genes including DHX58, HERC6, RTP4, IFI44, STAT2, MX2, STAT 1, IRF9, CXCL10, IFIT2, USP18, ISG15, OASL2, IFIT1, RSAD2, IRF7, DDX58, and IFIT3 may be involved in the progression of HD. Meanwhile, we found a new regulatory relationship between miRNA-124, STAT3 and RNASE4. All these findings can shed light on the complex pathogenesis of HD and provide a potential novel therapeutic strategy for patients with HD, but the bioinformatics findings obtained in this study require further conformation via experimental studies.

\section{Acknowledgements}

The present study was supported by the National Natural Science Foundation of China (no. 81371271), and was also sponsored by 'Liaoning BaiQianWan Talents Program'.

\section{References}

1. A novel gene containing a trinucleotide repeat that is expanded and unstable on Huntington's disease chromosomes. The Huntington's Disease Collaborative Research Group. Cell 72: 971-983, 1993

2. Sinha M,Mukhopadhyay S and Bhattacharyya NP: Mechanism(s) of alteration of Micro RNA expressions in huntington's disease and their possible contributions to the observed cellular and molecular dysfunctions in the disease. Neuromolecular Med 14 221-243, 2012.

3. Azambuja MJ, Haddad MS, Radanovic M, Barbosa ER and Mansur LL: Semantic, phonologic, and verb fluency in Huntington's disease. Dement Neuropsychol 1: 381-385, 2007.

4. Tabrizi SJ, Langbehn DR, Leavitt BR, Roos RA, Durr A, Craufurd D, Kennard C, Hicks SL, Fox NC, Scahill RI, et al: Biological and clinical manifestations of Huntington's disease in the longitudinal TRACK-HD study: Cross-sectional analysis of baseline data. Lancet Neurol 8: 791-801, 2009.

5. Orford K, Crockett C, Jensen JP, Weissman AM and Byers SW: Serine phosphorylation-regulated ubiquitination and degradation of beta-catenin. J Biol Chem 272: 24735-24738, 1997.
6. Seredenina $\mathrm{T}$ and Luthi-Carter R: What have we learned from gene expression profiles in Huntington's disease? Neurobiol Dis 45: 83-98, 2012.

7. Cong SY, Pepers BA, Evert BO, Rubinsztein DC, Roos RA, van Ommen GJ and Dorsman JC: Mutant huntingtin represses CBP, but not $\mathrm{p} 300$, by binding and protein degradation. Mol Cell Neurosci 30: 12-23, 2005.

8. Bae BI, Xu H, Igarashi S, Fujimuro M, Agrawal N, Taya Y, Hayward SD, Moran TH, Montell C, Ross CA, et al: p53 mediates cellular dysfunction and behavioral abnormalities in Huntington's disease. Neuron 47: 29-41, 2005.

9. Dunah AW, Jeong H, Griffin A, Kim YM, Standaert DG, Hersch SM, Mouradian MM, Young AB, Tanese N and Krainc D: Sp1 and TAFII130 transcriptional activity disrupted in early Huntington's disease. Science 296: 2238-2243, 2002.

10. Li JL, Hayden M, Almqvist EW, Brinkman RR, Durr A, Dodé C Morrison PJ, Suchowersky O, Ross CA, Margolis RL, et al: A genome scan for modifiers of age at onset in Huntington's disease: The HD MAPS study. Am J Hum Genet 73: 682-687, 2003.

11. Sinha M, Ghose J and Bhattarcharyya NP: Micro RNA-214,-150,-146a and-125b target Huntingtin gene. RNA Biol 8: 1005-1021, 2011

12. Santiago JA and Potashkin JA: A network approach to clinical intervention in neurodegenerative diseases. Trends Mol Med 20: 694-703, 2014

13. Nguyen TP, Caberlotto L, Morine MJ and Priami C: Network analysis of neurodegenerative disease highlights a role of Toll-like receptor signaling. Biomed Res Int 2014: 686505, 2014.

14. Hofmann-Apitius M, Ball G, Gebel S, Bagewadi S, de Bono B, Schneider R, Page M, Kodamullil AT, Younesi E, Ebeling C, et al: Bioinformatics mining and modeling methods for the identification of disease mechanisms in neurodegenerative disorders. Int J Mol Sci 16: 29179-29206, 2015.

15. Sadri-Vakili G, Bouzou B, Benn CL, Kim MO, Chawla P, Overland RP, Glajch KE, Xia E, Qiu Z, Hersch SM, et al: Histones associated with downregulated genes are hypo-acetylated in Huntington's disease models. Hum Mol Genet 16: 1293-1306, 2007.

16. Smyth GK: Limma: Linear models for microarray data. 2005.

17. Guo W and Rao MB: On control of the false discovery rate under no assumption of dependency. J Statistical Plan Inference 138: 3176-3188, 2008.

18. Huang Da W, Sherman BT and Lempicki RA: Systematic and integrative analysis of large gene lists using DAVID bioinformatics resources. Nat Protocols 4: 44-57, 2009.

19. Carbon S, Ireland A, Mungall CJ, Shu S, Marshall B and Lewis S; AmiGO Hub; Web Presence Working Group: AmiGO: Online access to ontology and annotation data. Bioinformatics 25: 288-289, 2009.

20. Kanehisa M, Araki M, Goto S, Hattori M, Hirakawa M, Itoh M, Katayama T, Kawashima S, Okuda S, Tokimatsu T and Yamanishi Y: KEGG for linking genomes to life and the environment. Nucleic Acids Res 36 (Database Issue): D480-D484, 2008.

21. Szklarczyk D, Franceschini A, Kuhn M, Simonovic M, Roth A, Minguez P, Doerks T, Stark M, Muller J, Bork P, et al: The STRING database in 2011: Functional interaction networks of proteins, globally integrated and scored. Nucleic Acids Res 39 (Database Issue): D561-D568, 2011.

22. Smoot ME, Ono K, Ruscheinski J, Wang PL and Ideker T: Cytoscape 2.8: New features for data integration and network visualization. Bioinformatics 27: 431-432, 2011.

23. Bader GD and Hogue CW: An automated method for finding molecular complexes in large protein interaction networks. BMC Bioinformatics 4: 2, 2003.

24. Lewis BP, Burge CB and Bartel DP: Conserved seed pairing, often flanked by adenosines, indicates that thousands of human genes are microRNA targets. Cell 120: 15-20, 2005.

25. Wingender E: The TRANSFAC project as an example of framework technology that supports the analysis of genomic regulation. Brief Bioinform 9: 326-332, 2008.

26. Zhang JF, Chen Y, Lin GS, Zhang JD, Tang WL, Huang JH, Chen JS, Wang XF and Lin ZX: High IFIT1 expression predicts improved clinical outcome, and IFIT1 along with MGMT more accurately predicts prognosis in newly diagnosed glioblastoma. Hum Pathol 52: 136-144, 2016.

27. Squitieri F, Cannella M, Sgarbi G, Maglione V, Falleni A, Lenzi P, Baracca A, Cislaghi G, Saft C, Ragona G, et al: Severe ultrastructural mitochondrial changes in lymphoblasts homozygous for Huntington disease mutation. Mech Ageing Dev 127: 217-220, 2006. 
28. Bayram-Weston Z, Stone TC, Giles P, Elliston L, Janghra N, Higgs GV, Holmans PA, Dunnett SB, Brooks SP and Jones L: Similar striatal gene expression profiles in the striatum of the YAC128 and HdhQ150 mouse models of Huntington's disease are not reflected in mutant Huntingtin inclusion prevalence. BMC Genomics 16: 1079, 2015.

29. Jordanovski D, Herwartz C, Pawlowski A, Taute S, Frommolt P and Steger G: The hypoxia-inducible transcription factor ZNF395 is controlled by I $\kappa$ B kinase-signaling and activates genes involved in the innate immune response and cancer. PLoS One 8: e74911, 2013.

30. Ellrichmann G, Reick C, Saft C and Linker RA: The role of the immune system in Huntington's disease. Clin Dev Immunol 2013 541259, 2013.

31. Zhu J,Zhang Y, Ghosh A, Cuevas RA, Forero A, Dhar J, Ibsen MS Schmid-Burgk JL, Schmidt T, Ganapathiraju MK et al: Antiviral activity of human OASL protein is mediated by enhancing signaling of the RIG-I RNA sensor. Immunity 40 : 936-948, 2014

32. Kwan W, Träger U, Davalos D, Chou A, Bouchard J, Andre R, Miller A, Weiss A, Giorgini F, Cheah C, et al: Mutant huntingtin impairs immune cell migration in Huntington disease. J Clin Invest 122: 4737-4747, 2012.

33. McDermott JE, Archuleta M, Thrall BD, Adkins JN and Waters KM: Controlling the response: Predictive modeling of a highly central, pathogen-targeted core response module in macrophage activation. PLoS One 6: e14673, 2011.

34. MacNair L, Xiao S, Miletic D, Ghani M, Julien JP, Keith J, Zinman L, Rogaeva E and Robertson J: MTHFSD and DDX58 are novel RNA-binding proteins abnormally regulated in amyotrophic lateral sclerosis. Brain 139: 86-100, 2016.

35. Tauffenberger A, Chitramuthu BP, Bateman A, Bennett HP and Parker JA: Reduction of polyglutamine toxicity by TDP-43, FUS and progranulin in Huntington's disease models. Hum Mol Genet 22: 782-794, 2013 .

36. de Rivero Vaccari JP, Brand FJ III, Sedaghat C, Mash DC, Dietrich WD and Keane RW: RIG-1 receptor expression in the pathology of Alzheimer's disease. J Neuroinflam 11: 67, 2014.

37. Oudshoorn D, van Boheemen S, Sánchez-Aparicio MT, Rajsbaum R, Garcia-Sastre A and Versteeg GA: HERC6 is the main E3 ligase for global ISG15 conjugation in mouse cells. PLoS One 7: e29870, 2012.

38. Wang RG, Kaul M and Zhang DX: Interferon-stimulated gene 15 as a general marker for acute and chronic neuronal injuries. Sheng Li Xue Bao 64: 577-583, 2012.

39. Desai SD, Reed RE, Babu S and Lorio EA: ISG15 deregulates autophagy in genotoxin-treated ataxia telangiectasia cells. J Biol Chem 288: 2388-2402, 2013.

40. Nicolas CS, Amici M, Bortolotto ZA, Doherty A, Csaba Z, Fafouri A, Dournaud P, Gressens P, Collingridge GL and Peineau S: The role of JAK-STAT signaling within the CNS. JAKSTAT 2: e22925, 2013.
41. Fu MH, Li CL, Lin HL, Tsai SJ, Lai YY, Chang YF, Cheng PH, Chen CM and Yang SH: The potential regulatory mechanisms of miR-196a in Huntington's disease through bioinformatic analyses. PLoS One 10: e0137637, 2015.

42. Ruvkun G: The perfect storm of tiny RNAs. Nat Med 14: 1041-1045, 2008

43. Krichevsky AM, King KS, Donahue CP, Khrapko K and Kosik KS: A microRNA array reveals extensive regulation of microRNAs during brain development RNA 9: 1274-1281, 2003.

44. Berezikov E, Thuemmler F, van Laake LW, Kondova I, Bontrop R, Cuppen E and Plasterk RH: Diversity of microRNAs in human and chimpanzee brain. Nat Genet 38: 1375-1377, 2006.

45. Kozlowska E, Krzyzosiak WJ and Koscianska E: Regulation of huntingtin gene expression by miRNA-137,-214,-148a, and their respective isomiRs. Int J Mol Sci 14: 16999-17016, 2013.

46. Sinha M, Ghose J, Das E and Bhattarcharyya NP: Altered microRNAs in STHdh(Q111)/Hdh(Q111) cells: miR-146a targets TBP. Biochem Biophys Res Commun 396: 742-747, 2010.

47. Ban JJ, Chung JY, Lee M, Im W and Kim M: MicroRNA-27a reduces mutant hutingtin aggregation in an in vitro model of Huntington's disease. Biochem Biophys Res Commun 488: 316-321, 2017.

48. Sheng J, Luo C, Jiang Y, Hinds $\mathrm{PW}, \mathrm{Xu} \mathrm{Z}$ and $\mathrm{Hu}$ GF: Transcription of angiogenin and ribonuclease 4 is regulated by RNA polymerase III elements and a CCCTC binding factor (CTCF)-dependent intragenic chromatin loop. J Biol Chem 289: 12520-12534, 2014.

49. Li S, Sheng J, Hu JK, Yu W, Kishikawa H, Hu MG, Shima K, Wu D, Xu Z, Xin W, et al: Ribonuclease 4 protects neuron degeneration by promoting angiogenesis, neurogenesis, and neuronal survival under stress. Angiogenesis 16: 387-404, 2013.

50. Fay A, Misulovin Z, Li J, Schaaf CA, Gause M, Gilmour DS and Dorsett D: Cohesin selectively binds and regulates genes with paused RNA polymerase. Curr Biol 21: 1624-1634, 2011.

51. Li S, Yu W and Hu GF: Angiogenin inhibits nuclear translocation of apoptosis inducing factor in a Bcl-2-dependent manner. J Cell Physiol 227: 1639-1644, 2012.

52. Johnson R and Buckley NJ: Gene dysregulation in Huntington's disease: REST, microRNAs and beyond. Neuromolecular Med 11: 183-199, 2009.

53. Das E, Jana NR and Bhattacharyya NP: MicroRNA-124 targets CCNA2 and regulates cell cycle in STHdh(Q111)/Hdh(Q111) cells. Biochem Biophys Res Commun 437: 217-224, 2013.

54. Ben Haim L, Ceyzériat K, Carrillo-de Sauvage MA, Aubry F, Auregan G, Guillermier M, Ruiz M, Petit F, Houitte D, Faivre E, et al: The JAK/STAT3 pathway is a common inducer of astrocyte reactivity in Alzheimer's and Huntington's diseases. J Neurosci 35: 2817-2829, 2015.

(c) (i) (5) This work is licensed under a Creative Commons cc) $\mathrm{EY}$ NG $\mathrm{ND}$ Attribution-NonCommercial-NoDerivatives 4.0 International (CC BY-NC-ND 4.0) License. 